\title{
Le traitement de l'hæmonchose et de l'œesophagostomose ovines par les agents tensio-actifs (alkylsulfates de soude)
}

\author{
par S. GRETILLAT et R. DAUMAS
}

Nous avons montré tout dernièrement l'intérêt que pouvaient présenter les corps tensio-actifs employés comme anthelminthiques dans la lutte contre les trématodes du rumen des bovidés.

En raison de son prix de revient extrêmement bas, ce nouveau procédé méritait d'être essayé dans le traitement d'autres helminthiases des animaux domestiques.

Le parasitisme gastro-intestinal étant chaque année la cause de nombreuses pertes parmi les petits élevages ovins malgaches, nous avons essayé les alkylsulfates de soude comme anthelminthiques chez le mouton.

Les helminthiases ovines les plus fréquentes dans la Grande Ile sont : l'haemonchose de la caillette à Haemonchus contortus (Rudolphi, 1803), l'cesophagostomose à Oesophagostomum colombianum Curtice, 1890, et Oesophagostomum venulosum (Rudolphi, 1809), et la moniéziose à Moniezia expansa (Rudolphi, 1810). Cette dernière affection parasitaire est surtout répandue dans les régions du sud de Madagascar.

\section{EXPERIMENTATION}

Nous avons tout d'abord procédé à des tests in vitro à l'aide d'une solution d'alkylsulfate de soude sur des helminthes fraîchement prélevés sur des cadavres et maintenus en survie dans l'eau physiologique à 9 p. 1000 .

Par la suite, des contrôles d'activité in vivo ont été faits sur trois ovins autopsiés quelques jours après leur vermifugation. Les résultats de ces essais nous ont permis de connaître l'ordre de grandeur de la dose curative.

Passant alors sur le plan de la pratique, nous avons essayé cet anthelminthique sur 80 ovins appartenant à des éleveurs autochtones des environs de Tananarive.

\section{Tests d'activité in vitro}

- Sur H. contortus :

In vitro une solution à $1 / 1.000$ d'alkylsulfate de soude tue ces helminthes en 2 à 3 heures.

Une solution à $1 / 3.000$ détruit ces parasites en 5 heures.

\section{- Sur Oesophagostomum colombianum :}

L'activité in vitro des mêmes solutions a un effet un peu plus rapide que sur $H$. contortus.

\section{Essais in vivo}

Réalisés sur trois ovins parasités massivement à $H$. contortus et Oesophagostum sp. (ces animaux ont été choisis après examen coprologique préliminaire).

Le produit (solution aqueuse d'alkylsulfate de soude titrant 10,5 p. 100 de produit pur) est administré à la sonde cesophagienne pour éviter toute perte de vermifuge. Quelques gouttes de produit antimousse à base de silicones sont ajoutées pour supprimer l'effet moussant de l'alkylsulfate de soude.

Ovin $n^{\circ} 1$ : Dose administrée : 0,04 gramme de produit pur par kilogramme de poids.

Autopsie : Quelques $H$. contortus encore vivants dans la caillette. Nombreux cesophagostomes dans le gros intestin.

Ovins $n^{\text {os }} 2$ et 3 : Dose administrée : 0,06 gramme de produit pur par kilogramme de poids.

Autopsies faites six jours après le traitement pour le mouton $n^{\circ} 2,10$ jours après la vermifugation pour le mouton $\mathrm{n}^{\mathbf{0}} 3$. Résultats : Absence totale de parasites dans le tube digestif de ces deux animaux.

La dose de 0,06 gramme de produit pur par kilogramme de poids d'animal s'étant révélée active contre $H$. contortus et Oesophagostum sp., nous décidons d'opérer sur une plus grande échelle en intervenant sur plusieurs dizaines d'ovins choisis dans les troupeaux d'éleveurs malgaches. 
TABLEAU I

ESSAIS DE VERUIITUGATION FAITS DANS LE CANTON D'AYBOANANA SUR 33 MOUTONS

\begin{tabular}{|c|c|c|c|c|c|c|c|c|}
\hline \multirow{2}{*}{$\begin{array}{l}\text { No du } \\
\text { mouton }\end{array}$} & \multicolumn{3}{|c|}{$\begin{array}{l}\text { Examen coprologique fait } \\
\text { avant le traitement }\end{array}$} & \multirow{2}{*}{$\begin{array}{l}\text { Dose } \\
\text { en ml }\end{array}$} & \multirow{2}{*}{$\begin{array}{l}\text { Observa- } \\
\text { tions }\end{array}$} & \multicolumn{3}{|c|}{$\begin{array}{l}\text { Examen coprologique fait } \\
\text { après le traitement }\end{array}$} \\
\hline & Haemonchus & $\begin{array}{l}\text { Oesopha- } \\
\text { gostomes }\end{array}$ & Divers & & & Heemonchus & $\begin{array}{l}\text { Oesopha- } \\
\text { gostomes }\end{array}$ & Divers \\
\hline $\begin{array}{l}43 \\
44 \\
45 \\
46 \\
47 \\
48 \\
49 \\
50 \\
51 \\
52 \\
53 \\
55 \\
56 \\
57 \\
58 \\
59 \\
60 \\
61 \\
62 \\
63 \\
65 \\
66 \\
67 \\
68 \\
69 \\
70 \\
71 \\
72 \\
74 \\
76 \\
77 \\
78 \\
79\end{array}$ & $\begin{array}{c}H+ \\
H+H \\
+++ \\
+ \\
++ \\
+ \\
+H+ \\
++ \\
+ \\
+H+ \\
+ \\
++ \\
+ \\
++ \\
++ \\
+H \\
+++ \\
++ \\
++ \\
+ \\
+ \\
++ \\
+ \\
++ \\
+ \\
+ \\
+ \\
+ \\
H \\
+ \\
+ \\
+ \\
+\end{array}$ & 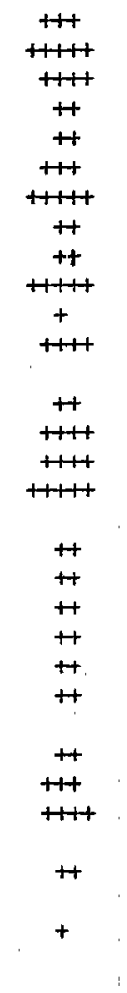 & $\begin{array}{c}\text { M. } \\
\text { S. H } \\
\text { S. } \\
\text { M. } \\
\text { M. } \\
\text { S.M. } \\
\text { S. }\end{array}$ & $\begin{array}{l}10 \\
12 \\
10 \\
15 \\
15 \\
15 \\
12 \\
15 \\
15 \\
15 \\
12 \\
10 \\
15 \\
12 \\
10 \\
15 \\
10 \\
12 \\
10 \\
10 \\
15 \\
15 \\
12 \\
15 \\
15 \\
15 \\
12 \\
15 \\
15 \\
10 \\
10 \\
12 \\
15\end{array}$ & $\bar{D}$. & $\begin{array}{l}+ \\
+ \\
+ \\
+ \\
+ \\
+ \\
+ \\
+ \\
+ \\
+ \\
+ \\
+ \\
+ \\
+ \\
+ \\
+ \\
+ \\
+ \\
+ \\
+ \\
+ \\
+ \\
+ \\
+\end{array}$ & $\begin{array}{c}+ \\
+ \\
+ \\
+ \\
++ \\
+ \\
+ \\
+ \\
+\end{array}$ & $\begin{array}{l}\text { S. } \\
\text {. }\end{array}$ \\
\hline
\end{tabular}

Légende des abréviations

$$
\begin{aligned}
& +++=\text { infestation massive, } \\
& ++=\text { infestation tres forte, } \\
& ++\quad \text { infestation forte, } \\
& +\quad=\text { infestation moyenne, } \\
& +\quad=\text { infestation faible. }
\end{aligned}
$$

\section{PROTOCOLE EXPÉRIMFNTAL}

Les animaux à vermifuger sont choisis parmi les antenais et les adultes, les femelles suitées et les gravides étant écartées de l'expérience (*).

Un examen coprologique est fait sur chaque ovin avant l'administration de l'anthelminthique en même temps qu'une marque numérotée lui est apposée à l'oreille s'il s'est révélé parasité. La densité et le genre de parasitisme est noté

(*) Nous tenons à remercier ici le Dr Savin, chef de la Circonscription d'Elevage de Tananarive pour l'aide qu'il nous a apportée dans ces essais de vermifugation.

$$
\begin{aligned}
& \text {DD}_{*}=\text { diarrhée, } \\
& \text {M. }_{*}=\text { Moniezia, } \\
& \text { S. }_{\text {. Strongles, }} \\
& \text { P. }_{\text {. }} \text { Paranphistomes. }
\end{aligned}
$$

pour chaque animal traité ainsi que la quantité de produit anthelminthique administrée.

L'efficacité du traitement est contrôlée par examen coprologique dans les semaines suivant le traitement.

Pour procéder à ces essais, nous avons utilisé un produit titrant 21 p. 100 de produit pur et dilué au moment de l'emploi dans une fois son volume d'eau. Afin d'éviter les risques d'asphyxie et de météorisation dus au pouvoir moussant de l'alkylsulfate de soude, nous ajoutons à la solution un produit antimousse à base de silicones 
TAEIEAU II

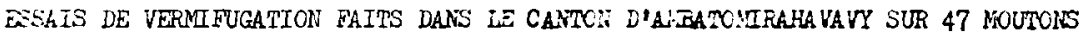

\begin{tabular}{|c|c|c|c|c|c|c|c|c|}
\hline \multirow{2}{*}{$\begin{array}{l}\text { Wo du } \\
\text { mouton }\end{array}$} & \multicolumn{3}{|c|}{$\begin{array}{l}\text { Examen coprologique fait } \\
\text { avant le traitement }\end{array}$} & \multirow{2}{*}{$\begin{array}{l}\text { Dose } \\
\text { en ml }\end{array}$} & \multirow{2}{*}{$\begin{array}{l}\text { Cbserve- } \\
\text { tions }\end{array}$} & \multicolumn{3}{|c|}{$\begin{array}{l}\text { Eramen coprologique fait } \\
\text { après le traitement }\end{array}$} \\
\hline & ilaemonchus & $\begin{array}{l}\text { Oesopha- } \\
\text { gostomes }\end{array}$ & Divers & & & Haemonohus & $\begin{array}{l}\text { Oesopha- } \\
\text { gostcomes }\end{array}$ & Divers \\
\hline $\begin{array}{l}501 \\
502 \\
503 \\
504 \\
505 \\
506 \\
507 \\
508 \\
509 \\
510 \\
511 \\
512 \\
513 \\
514 \\
515 \\
516 \\
517 \\
518 \\
520 \\
522 \\
523 \\
524 \\
525 \\
526 \\
527 \\
528 \\
529 \\
530 \\
531 \\
532 \\
533 \\
534 \\
535 \\
536 \\
537 \\
538 \\
539 \\
540 \\
541 \\
542 \\
543 \\
544 \\
545 \\
546 \\
547 \\
548 \\
549\end{array}$ & 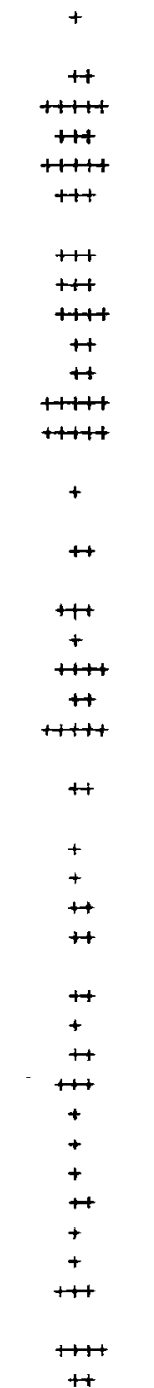 & 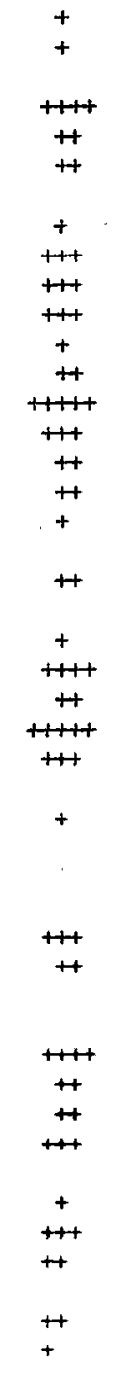 & 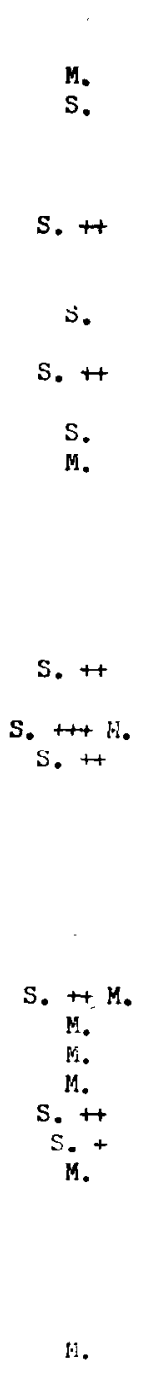 & $\begin{array}{l}30 \\
25 \\
20 \\
20 \\
20 \\
30 \\
30 \\
20 \\
25 \\
30 \\
30 \\
30 \\
30 \\
30 \\
20 \\
30 \\
30 \\
30 \\
30 \\
30 \\
30 \\
20 \\
25 \\
25 \\
30 \\
20 \\
20 \\
20 \\
20 \\
15 \\
15 \\
20 \\
15 \\
15 \\
15 \\
10 \\
25 \\
20 \\
25 \\
20 \\
15 \\
20 \\
25 \\
25 \\
25 \\
15 \\
20\end{array}$ & $\begin{array}{l}\bar{D} . \\
D .\end{array}$ & $\begin{array}{l}+ \\
+ \\
+\end{array}$ & $\begin{array}{l}+ \\
+ \\
+\end{array}$ & 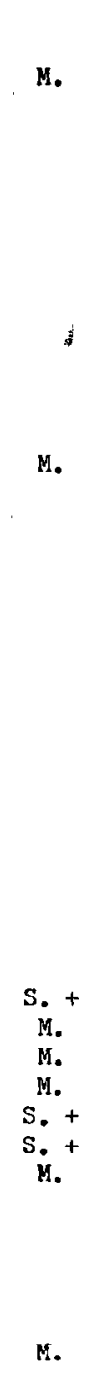 \\
\hline
\end{tabular}

Légende des àréviations

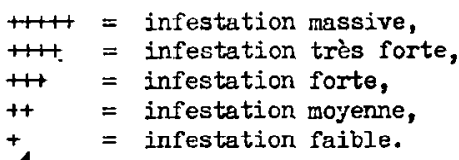

$H+H=$ infestation massive,

H. = infestation très forte,

$++\quad=$ infestation moyenne,

$+\quad=$ infestation faible.
J. = diarrhée,

H. = Soniezia,

S. = Etrongles,

P. = Faremphistanes. 
à raison de $10 \mathrm{ml}$ par litre de vermifuge (antimousse Shell).

L'administration de l'anthelminthique est faite au pistolet doseur.

Pour les examens coprologiques, nous avons choisi la méthode de Willis (liquides denses), très pratique en brousse et qui donne de très bons résultats danș le cas qui nous intéresse.

\section{Tableau $\mathbf{n}^{0}$ I}

\section{DISCUSSION}

La dose approximative par kilogramme de poids a été de 0,06 gramme de produit pur.

Des contrôles d'efficacité ont été faits 9 jours après le traitement anthelminthique.

Nous constatons au contrôle de fin de traitement une très nette diminution de la densité du parasitisme avec amélioration de l'état général de la plupart des animaux vermifugés. Cependant, certains ovins présentent encore dans leurs excréments de nombreux oufs d'helminthes.

Nous attribuons cet échec partiel à une mauvaise administration du vermifuge. L'animal, fen se débattant au cours du traitement, rejette souvent une partie de la solution anthelminthique.

\section{Tableau $\mathbf{n}^{0}$ II}

La dose par kilogramme de poids a été augmentée par rapport à l'expérience précédente. Elle est portée à $0,09 \mathrm{~g} / \mathrm{kg}$.

Les contrôles d'efficacité sont faits 20 jours après le traitement.

Comme on peut facilement s'en rendre compte en examinant le tableau $\mathrm{n}^{\circ}$ II, les résultats sont très satisfaisants.

L'efficacité est pratiquement totale vis-à-vis des Haemonchus et des œesophagostomes.

De l'avis même des éleveurs, l'état général des animaux s'est considérablement amélioré.

\section{CONCLUSION}

Des résultats que nous avons obtenus, il ressort qu'une solution d'alkylsulfate de soude s'avère être un très bon anthelminthique dans la lutte contre l'haemonchose et l'csophagostomose ovines. Par contre, ce produit semble beaucoup moins intéressant dans le traitement de la monieziose ovine.

Pratiquement, les doses à utiliser sur des moutons adultes ou des antenais sont, suivant le poids des animaux, de 15 à $30 \mathrm{ml}$ de solution d'alkylsulfate de soude titrant 10,5 p.' 100 de produit pur.

En utilisant une dose de 0,09 gramme de produit pur par kilogramme de poids, on obtient une bonne vermifugation sans aucun risque d'accident, la dose toxique pour les ovins étant comme nous l'avons indiqué dernièrement, de 0,43 à 0,45 gramme par kilogramme de poids.

Nous tenons cependant à signaler qu'il y a lieu d'éviter, au cours de la vermifugation, toute introduction de produit dans la trachée de l'animal.

Laboratoire central de l'Elevage, Services de parasitologie et de chimie, Tananarive.

\section{BIBLIOGRAPHIE}

Daumas (R.) et GrÉtillat (S.) (1958). Les alkyl sulfates de soude : nouveaux anthelminthiques. (Ann. Pharmac. Franc., sous presse).

GrÉtillat (S.) et Daumas (R.) (1958). - Mise au point d'un nouveau procédé de lutte anthel minthique au moyen d'agents tensio-actifs (Alkylsulfates de sodium) utilisés dans le traitement de la gastrothylose bovine à Madagascar. Rev. Elev. Méd. vét. Pays trop., 1958, 11 (3), 257-64. 


\section{SUMMARY}

\section{Treatment of Hæmonchus and Oesophagostomum infestations in sheep by high surface tension agents (sodium alkylsulphates)}

The authors tested the efficacity of certain high surface tension agents, the alkylsulphates of sodium in the control against certain ovine helminthiases. Previously they had shown the value of these drugs which are inexpensive and of low toxicity in gastrothylosis of cattle. In vitro trials and extensive in vivo experiments on native sheep in Madagascar showed that these drugs gave very good results in Haemonchus and Oesophagostomum infestations. The recommended dose is $0.09 \mathrm{~g}$. $/$ $\mathrm{kg}$ l.w. The toxic dose is from $0.43-0.45 \mathrm{~g} . / \mathrm{kg}$. To the solution of $10.5 \%$ pure sodium alkylsulphate is added $1 \%$ of an anti-frothing mixture.

\section{RESUMEN}

\section{El tratamiento de la hemonchomosis y de la esofagostomosis ovinas por los agentes tensio-activos (alkylsulfatos de sodio)}

Los autores examinan la eficacia de los agentes tensio-activos, los alkylsulfatos de sodio, en la lucha contra ciertas helmintiasis ovinas ; anteriomente, ellos habian mostrado el valor de estos productos muy poco tóxicos y poco costosos contra la gastrofilosis bovina. Despues de los ensayos " in vitro" $\mathrm{e}$ " in vivo" seguidos de una expemirentación en gran escala en los rebaños de ganaderos de Madagascar, han comprobado que el producto utilizado daba muy buenos resultados contra la hemonchosis y la esof gostomosis ; la dosis preconizada es de 0,09 gramos del producto puro por kilogramo de peso vivo, la dosis tóxica comienza desde 0,43 ó 0,45 gramos por kilogramo. Utilizan una solucion de alkylsulfato de sodio al 10,5 p. 100 de producto puro adicionado de un producto antiespumoso al 1 p. 100. 